\title{
On the sharpness of the Rüssmann estimates
}

\author{
Jordi-Lluís Figueras* \\ Department of Mathematics \\ Uppsala University \\ Box 480, 75106 Uppsala (Sweden). \\ Alex $\operatorname{Haro}^{\dagger}$ \\ Departament de Matemàtiques i Informàtica \\ Universitat de Barcelona \\ Gran Via 585, 08007 Barcelona (Spain).
}

\author{
Alejandro Luque \\ Instituto de Ciencias Matemáticas \\ Consejo Superior de Investigaciones Científicas \\ C/ Nicolás Cabrera 13-15, 28049 Madrid (Spain).
}

17th November 2018

\begin{abstract}
Estimating the norm of the solution of the linear difference equation $u(\theta)-u(\theta+\omega)=$ $v(\theta)$ plays a fundamental role in KAM theory. Optimal (in certain sense) estimates for the solution of this equation were provided by Rüssmann in the mid 70's. The aim of this paper is to compare the sharpness of these classic estimates with more specific estimates obtained with the help of the computer. We perform several experiments to quantify the improvement obtained when using computer assisted estimates. By comparing these estimates with the actual norm of the solution, we can analyze the different sources of overestimation, thus encouraging future improvements.
\end{abstract}

Keywords: KAM theory; cohomologic equations; Rüssmann estimates; small divisors.

\footnotetext{
*figueras@math.uu.se

†alex@maia.ub.es

‡luque@icmat.es
} 


\section{Introduction}

Given a zero-average periodic function $v: \mathbb{T}^{n} \rightarrow \mathbb{C}$ and a vector $\omega \in \mathbb{R}^{n}$, we consider the linear difference equation

$$
u(\theta)-u(\theta+\omega)=v(\theta) .
$$

This cohomological equation plays a central role in KAM theory, and arises for example in the proof of the existence of invariant tori for symplectic maps (see [15, 19] and [1, 2, 3, 7, 12, 16] for other contexts). A typical KAM scheme consists in performing a Newton-like iteration process such that Equation (1) has to be solved at every iteration step. The convergence of the procedure in a certain scale of Banach spaces of regular functions (endowed with a suitable norm) is ensured by estimating the norm of the solution of (1) in such scale. The optimality of these estimates is crucial for applying the KAM theorem in specific examples and determining the size of the perturbation for which invariant tori persist ${ }^{1}$. These optimal estimates were provided by Rüssmann in the celebrated paper [20] (see [18] for the study of the analogous linear differential equation).

A computer-assisted methodology to apply the KAM theory in particular problems, based on the so-called a posteriori approach, has been recently presented in [10]. A direct application of this methodology permitted to prove the existence of the golden invariant curve for the standard map up to a perturbation parameter $\varepsilon=0.9716$, which is less than $0.004 \%$ below the breakdown threshold observed numerically (see [11] for a semi-numeric criteria and [4, 8, 9] for previous computer assisted proofs). One of the technical improvements presented in [10] is the use of sharper Rüssmann estimates, obtained with the help of the computer. The idea behind these estimates is to compute explicitly a finite number of divisors, and use Diophantine properties to control the remaining ones.

The aim of this paper is to present a suitable and detailed illustration of the improved Rüssmann estimates used in [10], and to compare this estimates both with the classic Rüssmann ones and with a good numerical approximation of the solution. To this end, we use suitably chosen test functions and discuss the dependence on the different parameters of the problem. Hence, we quantify the improvement of the computer assisted estimates with respect to the classic ones, and also measure the error with respect to the actual norm.

The content of the paper is organized as follows. In Section 2 we formalize the problem outlined above. After recalling some elementary notation on analytic periodic functions and norms, we revisit the Rüssmann estimates to control the regularity of the solutions of Equation (1). We introduce some convenient notation to analyze the different sources of overestimation. Finally, we include some useful estimates to control the norm of an analytic function using discrete Fourier transform. In Section 3 we describe a methodology to enclose the norm of an analytic function. We illustrate quantitatively that the computation of a finite number of small divisors is enough to numerically capture the analytic norm of the solutions. In Section 4 we present some numerical studies of the behavior of the norm of the solutions of Equation (1), and we compare it with the Rüssmann estimates. Due to the large number of parameters in the problem, we consider several situations separately. In Section 5 we summarize the conclusions of our study, and present some conjectures derived from the experiments in Section 4, thus encouraging future research.

\footnotetext{
${ }^{1}$ The interested reader is referred to Section 1.4 in [6] for a brief history and references of the application of KAM theory, and to [5, 13, 14] for computer assisted proofs in problems of celestial mechanics.
} 


\section{Notation and basic results}

To present the previous statements in a precise way, we need to introduce some notation regarding analytic functions on the torus. We use standard notation for the real torus $\mathbb{T}^{n}=\mathbb{R}^{n} / \mathbb{Z}^{n}$ and the closed complex disk $\overline{\mathbb{D}}=\{z \in \mathbb{C}:|z| \leq 1\}$. A complex strip of $\mathbb{T}^{n}$ of width $\rho>0$ is defined as

$$
\mathbb{T}_{\rho}^{n}=\left\{\theta \in \mathbb{C}^{n} / \mathbb{Z}^{n}:\left|\operatorname{Im} \theta_{i}\right|<\rho, i=1, \ldots, n\right\} .
$$

We consider analytic functions $u: \mathbb{T}_{\rho}^{n} \rightarrow \mathbb{C}$ continuous up to the boundary of $\mathbb{T}_{\rho}^{n}$, and endow these functions with the norm

$$
\|u\|_{\rho}=\sup _{\theta \in \mathbb{T}_{\rho}^{n}}|u(\theta)| .
$$

Moreover, we denote the Fourier expansion by

$$
u(\theta)=\sum_{k \in \mathbb{Z}^{n}} \hat{u}_{k} \mathrm{e}^{2 \pi \mathrm{i} k \cdot \theta}, \quad \hat{u}_{k}=\int_{[0,1]^{n}} u(\theta) \mathrm{e}^{-2 \pi \mathrm{i} k \cdot \theta} \mathrm{d} \theta .
$$

\subsection{Estimates for the solution of the cohomological equation}

If $v$ is a function with zero average $\left(\hat{v}_{0}=0\right)$ and $\omega$ is ergodic, then Equation (1) has a formal solution $u:=\mathcal{R} v$ given by

$$
\mathcal{R} v(\theta)=\sum_{k \in \mathbb{Z}^{n} \backslash\{0\}} \hat{u}_{k} \mathrm{e}^{2 \pi \mathrm{i} k \cdot \theta}, \quad \hat{u}_{k}=\frac{\hat{v}_{k}}{1-\mathrm{e}^{2 \pi \mathrm{i} k \cdot \omega}} .
$$

Notice that all solutions of Equation (1) differ by a constant, since $\hat{u}_{0}$ is free.

Due to the effect of the small divisors $1-\mathrm{e}^{2 \pi \mathrm{i} k \cdot \omega}$ in Equation (4), it is well known that ergodicity is not enough to ensure regularity of the solution. Regularity is obtained by imposing non-resonant conditions on $\omega$. In this paper, we consider the following classic condition:

Definition 1. Given $\gamma>0$ and $\tau \geq n$, we say that $\omega \in \mathbb{R}^{n}$ is a $(\gamma, \tau)$-Diophantine vector of frequencies if

$$
|k \cdot \omega-m| \geq \gamma|k|_{1}^{-\tau}, \quad \forall k \in \mathbb{Z}^{n} \backslash\{0\}, m \in \mathbb{Z},
$$

where $|k|_{1}=\sum_{i=1}^{n}\left|k_{i}\right|$.

Notice that if $\omega$ is $(\gamma, \tau)$-Diophantine, then it is also $(\tilde{\gamma}, \tilde{\tau})$-Diophantine with $\tilde{\gamma} \leq \gamma$ and $\tilde{\tau} \geq \tau$. The set of $(\gamma, \tau)$-Diophantine vectors has positive Lebesgue measure if $\tau>n$.

The following result provides sufficient conditions to control, with explicit estimates, the norm of the solution of Equation (1):

Theorem 1. Let $\omega \in \mathbb{R}^{n}$ be $a(\gamma, \tau)$-Diophantine frequency vector, for certain $\gamma>0$ and $\tau \geq n$. Then, for any analytic function $v: \mathbb{T}_{\rho}^{n} \rightarrow \mathbb{C}$, with $\|v\|_{\rho}<\infty$ and $\rho>0$, there exists a unique zero-average analytic solution $u: \mathbb{T}_{\rho}^{n} \rightarrow \mathbb{C}$ of $(1)$, with $u=\mathcal{R} v$. Moreover, given $L \in \mathbb{N}$, for any $0<\delta<\rho$ we have

$$
\|u\|_{\rho-\delta} \leq \frac{c_{R}(\delta)}{\gamma \delta^{\tau}}\|v\|_{\rho}
$$


where

$$
c_{R}(\delta)=\sqrt{\gamma^{2} \delta^{2 \tau} 2^{n} \sum_{0<|k|_{1} \leq L} \frac{\mathrm{e}^{-4 \pi|k|_{1} \delta}}{4|\sin (\pi k \cdot \omega)|^{2}}+2^{n-3} \zeta\left(2,2^{\tau}\right)(2 \pi)^{-2 \tau} \int_{4 \pi \delta(L+1)}^{\infty} u^{2 \tau} e^{-u} \mathrm{~d} u},
$$

and $\zeta(a, b)=\sum_{j \geq 0}(b+j)^{-a}$ is the Hurwitz zeta function.

Sketch of the proof. The proof of this result, fully discussed in [10], follows using the same arguments originated in [18, 20], but with an eye in the feasibility of computing rigorous upper bounds of finite sums. For the sake of completeness, we discuss the sketch of the proof in order to point out several inequalities which will be analyzed later. We omit the details that are not related with the numerical explorations presented in this paper.

We note that the divisors in the expression (4) are written as

$$
\left|1-\mathrm{e}^{2 \pi \mathrm{i} k \cdot \omega}\right|=2|\sin (\pi k \cdot \omega)| \text {. }
$$

Then, the norm of $\mathcal{R} v$ is controlled as

$$
\|\mathcal{R} v\|_{\rho-\delta} \leq \sum_{k \in \mathbb{Z}^{n} \backslash\{0\}} \frac{\left|\hat{v}_{k}\right| \mathrm{e}^{2 \pi|k|_{1}(\rho-\delta)}}{2|\sin (\pi k \cdot \omega)|}
$$

and using Cauchy-Schwarz inequality we obtain

$$
\|\mathcal{R} v\|_{\rho-\delta} \leq\left(\sum_{k \in \mathbb{Z}^{n} \backslash\{0\}}\left|\hat{v}_{k}\right|^{2} \mathrm{e}^{4 \pi|k|_{1} \rho}\right)^{1 / 2}\left(\sum_{k \in \mathbb{Z}^{n} \backslash\{0\}} \frac{\mathrm{e}^{-4 \pi|k|_{1} \delta}}{4|\sin (\pi k \cdot \omega)|^{2}}\right)^{1 / 2} .
$$

On the one hand, the first term in $(8)$ is bounded by

$$
\sum_{k \in \mathbb{Z}^{n} \backslash\{0\}}\left|\hat{v}_{k}\right|^{2} \mathrm{e}^{4 \pi|k|_{1} \rho} \leq \sum_{k \in \mathbb{Z}^{n}}\left|\hat{v}_{k}\right|^{2} \mathrm{e}^{4 \pi|k|_{1} \rho} \leq 2^{n}\|v\|_{\rho}^{2}
$$

(see details in [18]), and on the other hand, we split the second term as

$$
\sum_{k \in \mathbb{Z}^{n} \backslash\{0\}} \frac{\mathrm{e}^{-4 \pi|k|_{1} \delta}}{4|\sin (\pi k \cdot \omega)|^{2}}=\sum_{0<|k|_{1} \leq L} \frac{\mathrm{e}^{-4 \pi|k|_{1} \delta}}{4|\sin (\pi k \cdot \omega)|^{2}}+\sum_{|k|_{1}>L} \frac{\mathrm{e}^{-4 \pi|k|_{1} \delta}}{4|\sin (\pi k \cdot \omega)|^{2}} .
$$

The finite sum of the elements $0<|k|_{1} \leq L$ can be evaluated for the selected frequency vector $\omega$. Then, the tail is controlled using the standard Rüssmann argument.

We highlight the following cases:

- Classic Rüssmann estimates: In this case, $c_{R}(\delta)$ is uniformly estimated by a constant $c_{R}^{0}$ independent of $\delta$. Indeed, for $L=0$ we have

$$
\begin{aligned}
c_{R}(\delta) & =\left(2^{n-3} \zeta\left(2,2^{\tau}\right)(2 \pi)^{-2 \tau} \int_{4 \pi \delta}^{\infty} u^{2 \tau} e^{-u} \mathrm{~d} u\right)^{1 / 2} \\
& \leq\left(2^{n-3} \zeta\left(2,2^{\tau}\right)(2 \pi)^{-2 \tau} \Gamma(2 \tau+1)\right)^{1 / 2}=: c_{R}^{0} .
\end{aligned}
$$

This is the classic Rüssmann constant (see [18, 20]). 
- Ad hoc Rüssmann estimates: Using a computer it is standard to obtain a sharp rigorous upper bound of the expression for $c_{R}(\delta)$ given in Equation (6). To this end, we enclose $\omega$ with an interval vector $\varpi$ (such that $\sin (\pi k \cdot \omega) \neq 0$ for every $\omega \in \varpi$ and for every $\left.|k|_{1} \leq L\right)$, and we rigorously enclose the finite sum for $0<|k|_{1} \leq L$ using interval arithmetics. Moreover, we consider upper bounds of the integral in the tail using that, if $y>x$,

$$
\int_{y}^{\infty} u^{x} e^{-u} \mathrm{~d} u \leq \frac{y}{y-x} y^{x} e^{-y} .
$$

Applying this last estimate requires to take $L$ such that $2 \pi \delta(L+1)>\tau$. This approach has been used in [10], where we refer to the reader for additional implementation details.

Remark 1. The estimates presented above are optimal in the sense of the asymptotic dependence of the divisor $\gamma \delta^{\tau}$ (former works in the literature used the much pessimistic factor $\gamma \delta^{n+\tau}$ ). This follows by studying functions with a single harmonic $v_{k}(\theta):=\mathrm{e}^{2 \pi \mathrm{i} k \cdot \theta}$. A direct computation yields the estimate

$$
\left\|\mathcal{R} v_{k}\right\|_{\rho-\delta} \leq \frac{\mathrm{e}^{2 \pi|k|_{1}(\rho-\delta)}}{2|\sin (\pi k \cdot \omega)|} \leq \frac{1}{4 \gamma}|k|_{1}^{\tau} \mathrm{e}^{-2 \pi|k|_{1} \delta}\left\|v_{k}\right\|_{\rho} \leq \frac{1}{4 \gamma}\left(\frac{\tau}{2 \pi \mathrm{e} \delta}\right)^{\tau}\left\|v_{k}\right\|_{\rho} .
$$

This computation also shows that Rüssmann estimates may provide a large overestimation for a fixed function in the above family. As Rüssmann himself observed in [18], the estimate in (13) seems to be the best possible estimate in the class of classic estimates, and he was able to obtain such an estimate for the case $n=1$ combining his approach with the theory of continued fractions. As far as we know, this question still remains open for $n>1$.

Motivated by the above discussion, we introduce a functional $F_{\rho, \delta, \omega}$ acting on $v$ as follows

$$
F_{\rho, \delta, \omega} v:=\frac{\|\mathcal{R} v\|_{\rho-\delta}}{\|v\|_{\rho}} \leq\|\mathcal{R}\|_{\rho, \rho-\delta},
$$

where $\|\mathcal{R}\|_{\rho, \rho-\delta}$ is the norm of the operator $\mathcal{R}$ acting between the corresponding spaces. Then, it turns out that the Rüssmann estimates provide upper bounds for the image of the previous functional:

$$
F_{\rho, \delta, \omega} v \leq \frac{c_{R}(\delta)}{\gamma \delta^{\tau}} \leq \frac{c_{R}^{0}}{\gamma \delta^{\tau}}
$$

In Section 4 we are going to study numerically the sharpness of these two inequalities as a function of $\rho, \delta, \omega$, and $v$.

\subsection{Analyzing the different sources of overestimation}

Assume that we take $L$ large enough in such a way that the contribution of the tail in Equation (6) can be neglected. In this case, it is interesting to analyze the different sources of the overestimation given by Theorem 1 .

We denote

$$
1 \leq I_{R}:=\frac{c_{R}(\delta)}{\gamma \delta^{\tau}} \frac{1}{F_{\rho, \delta, \omega} v}=\left(2^{n-2} \sum_{k \in \mathbb{Z}^{n} \backslash\{0\}} \frac{\mathrm{e}^{-4 \pi|k|_{1} \delta}}{|\sin (\pi k \cdot \omega)|^{2}}\right)^{1 / 2} \frac{\|v\|_{\rho}}{\|\mathcal{R} v\|_{\rho-\delta}},
$$


which stands for the overestimation produced by the ad hoc Rüssmann estimates.

It is clear that the expression of $I_{R}$ breaks down in terms of three different sources of overestimation as $I_{R}=I_{1} I_{2} I_{3}$, where

$$
\begin{aligned}
I_{1} & :=\frac{\sum_{k \in \mathbb{Z}^{n} \backslash\{0\}} \frac{\left|\hat{v}_{k}\right| \mathrm{e}^{2 \pi|k|_{1}(\rho-\delta)}}{2|\sin (\pi k \cdot \omega)|}}{\|\mathcal{R} v\|_{\rho-\delta}}, \\
I_{2} & :=\frac{\sqrt{\left(\sum_{k \in \mathbb{Z}^{n} \backslash\{0\}}\left|\hat{v}_{k}\right|^{2} \mathrm{e}^{4 \pi|k|_{1} \rho}\right)\left(\sum_{k \in \mathbb{Z}^{n} \backslash\{0\}} \frac{\mathrm{e}^{-4 \pi|k|} \delta}{4|\sin (\pi k \cdot \omega)|^{2}}\right)}}{\sum_{k \in \mathbb{Z}^{n} \backslash\{0\}} \frac{\left|\hat{v}_{k}\right| \mathrm{e}^{2 \pi|k|_{1}(\rho-\delta)}}{2|\sin (\pi k \cdot \omega)|}} \\
I_{3} & :=\frac{2^{n / 2}\|v\|_{\rho}}{\sqrt{\sum_{k \in \mathbb{Z}^{n} \backslash\{0\}}\left|\hat{v}_{k}\right|^{2} \mathrm{e}^{4 \pi|k|_{1} \rho}}} .
\end{aligned}
$$

Notice that $I_{1}, I_{2}, I_{3}$ correspond to inequalities (7), (8) and (9) respectively. Since

$$
\frac{\log \left(I_{1}\right)}{\log \left(I_{R}\right)}+\frac{\log \left(I_{2}\right)}{\log \left(I_{R}\right)}+\frac{\log \left(I_{3}\right)}{\log \left(I_{R}\right)}=1,
$$

we can make use of a color chart to represent the contribution (fraction in logarithmic scale) to each element to the total overestimation (see Figure 3 for an example).

Moreover, we observe that the expression in (16) also breaks down in two factors. On the one hand, the factor

$$
\frac{c_{R}(\delta)}{\gamma \delta^{\tau}}=\left(2^{n-2} \sum_{k \in \mathbb{Z}^{n} \backslash\{0\}} \frac{\mathrm{e}^{-4 \pi|k|_{1} \delta}}{|\sin (\pi k \cdot \omega)|^{2}}\right)^{1 / 2}
$$

is independent of $v$ and is decreasing with respect to $\delta$, and on the other hand, the factor

$$
\frac{1}{F_{\rho, \delta, \omega} v}=\frac{\|v\|_{\rho}}{\|\mathcal{R} v\|_{\rho-\delta}}
$$

is increasing with respect to $\delta$.

\subsection{Using discrete Fourier transform to approximate analytic functions}

Here, we briefly recall some explicit estimates presented in [10] that allows us to control the norm of an analytic function in terms of the norm of its discrete Fourier transform. Using this estimates we are able to rigorously validate the numerical computations using a finite number of Fourier coefficients.

We consider a sample of points on the regular grid of size $N=\left(N_{1}, \ldots, N_{n}\right) \in \mathbb{N}^{n}$

$$
\theta_{j}:=\left(\theta_{j_{1}}, \ldots, \theta_{j_{n}}\right)=\left(\frac{j_{1}}{N_{1}}, \ldots, \frac{j_{n}}{N_{n}}\right),
$$

where $j=\left(j_{1}, \ldots, j_{n}\right)$, with $0 \leq j_{\ell}<N_{\ell}$ and $1 \leq \ell \leq n$. This defines an $n$-dimensional sampling $\left\{u_{j}\right\}$, with $u_{j}=u\left(\theta_{j}\right)$. The integrals in Equation (3) are approximated using the discrete Fourier transform:

$$
\tilde{u}_{k}=\frac{1}{N_{1} \cdots N_{n}} \sum_{0 \leq j<N} u_{j} \mathrm{e}^{-2 \pi \mathrm{i} k \cdot \theta_{j}},
$$


where the sum runs over integer subindices $j \in \mathbb{Z}^{n}$ such that $0 \leq j_{\ell}<N_{\ell}$ for $\ell=1, \ldots, n$. Notice that $\tilde{u}_{k}$ is periodic with respect to the components $k_{1}, \ldots, k_{n}$ of $k$, with periods $N_{1}, \ldots, N_{n}$, respectively. The periodic function $u$ is approximated by the discrete Fourier approximation

$$
\tilde{u}(\theta)=\sum_{k \in \mathcal{I}_{N}} \tilde{u}_{k} \mathrm{e}^{2 \pi \mathrm{i} k \cdot \theta}
$$

where

$$
\mathcal{I}_{N}=\left\{k \in \mathbb{Z}^{n} \mid-\frac{N_{\ell}}{2} \leq k_{\ell}<\frac{N_{\ell}}{2}, 1 \leq \ell \leq n\right\} .
$$

is a finite set of multi-indices.

Theorem 2. Let $v: \mathbb{T}_{\tilde{\rho}}^{n} \rightarrow \mathbb{C}$ be an analytic function for $\tilde{\rho}>0$ and continuous up to the boundary. Let $\tilde{v}$ be the discrete Fourier approximation of $u$ in the regular grid of size $N=$ $\left(N_{1}, \ldots, N_{n}\right) \in \mathbb{N}^{n}$. Then

$$
\|\tilde{v}-v\|_{\rho} \leq C_{N}(\rho, \tilde{\rho})\|v\|_{\tilde{\rho}}
$$

for $0 \leq \rho<\tilde{\rho}$, where

$$
C_{N}(\rho, \tilde{\rho})=S_{N}^{* 1}(\rho, \tilde{\rho})+S_{N}^{* 2}(\rho, \tilde{\rho})+T_{N}(\rho, \tilde{\rho})
$$

is given by

$$
\begin{aligned}
& S_{N}^{* 1}(\rho, \tilde{\rho})=\prod_{\ell=1}^{n} \frac{1}{1-\mathrm{e}^{-2 \pi \tilde{\rho} N_{\ell}}} \sum_{\sigma \in\{-1,1\}^{n}} \prod_{\ell=1}^{n} \mathrm{e}^{\left(\sigma_{\ell}-1\right) \pi \tilde{\rho} N_{\ell}} \nu_{\ell}\left(\sigma_{\ell} \tilde{\rho}-\rho\right), \\
& \sigma \neq(1, \ldots, 1) \\
& S_{N}^{* 2}(\rho, \tilde{\rho})=\prod_{\ell=1}^{n} \frac{1}{1-\mathrm{e}^{-2 \pi \tilde{\rho} N_{\ell}}}\left(1-\prod_{\ell=1}^{n}\left(1-\mathrm{e}^{-2 \pi \tilde{\rho} N_{\ell}}\right)\right) \prod_{\ell=1}^{n} \nu_{\ell}(\tilde{\rho}-\rho)
\end{aligned}
$$

and

$$
T_{N}(\rho, \tilde{\rho})=\left(\frac{\mathrm{e}^{2 \pi(\tilde{\rho}-\rho)}+1}{\mathrm{e}^{2 \pi(\tilde{\rho}-\rho)}-1}\right)^{n}\left(1-\prod_{\ell=1}^{n}\left(1-\mu_{\ell}(\tilde{\rho}-\rho) e^{-\pi(\tilde{\rho}-\rho) N_{\ell}}\right)\right),
$$

with

$$
\nu_{\ell}(\delta)=\frac{\mathrm{e}^{2 \pi \delta}+1}{\mathrm{e}^{2 \pi \delta}-1}\left(1-\mu_{\ell}(\delta) \mathrm{e}^{-\pi \delta N_{\ell}}\right) \quad \text { and } \quad \mu_{\ell}(\delta)=\left\{\begin{array}{ll}
1 & \text { if } N_{\ell} \text { is even } \\
\frac{2 \mathrm{e}^{\pi \delta}}{\mathrm{e}^{2 \pi \delta}+1} & \text { if } N_{\ell} \text { is odd }
\end{array} .\right.
$$

Notice that $C_{N}(\rho, \tilde{\rho})$ satisfies $C_{N}(\rho, \tilde{\rho})=\mathcal{O}\left(\mathrm{e}^{-\pi(\tilde{\rho}-\rho) \min _{\ell} N_{\ell}}\right)$.

We refer the reader to [10] for the proof and implementation details. By combining Theorem 1 and Theorem 2 we obtain a direct way to control $F_{\rho, \delta, \omega} v$ for a given function $v$.

Corollary 1. Let $\omega \in \mathbb{R}^{n}$ be a $(\gamma, \tau)$-Diophantine frequency vector, for certain $\gamma>0$ and $\tau \geq n$. Let $v: \mathbb{T}_{\hat{\rho}}^{n} \rightarrow \mathbb{C}$ be an analytic function for $\hat{\rho}>0$. Then, for any $0<\delta \leq \rho<\tilde{\rho}<\hat{\rho}$ we have the following interval enclosure

$$
F_{\rho, \delta, \omega} v=\frac{\|\mathcal{R} v\|_{\rho-\delta}}{\|v\|_{\rho}} \in\left[\frac{\|\mathcal{R} \tilde{v}\|_{\rho-\delta}-\frac{c_{R}(\delta) C_{N}(\rho, \tilde{\rho})}{\gamma \delta^{\tau}}\|v\|_{\tilde{\rho}}}{\|\tilde{v}\|_{\rho}+C_{N}(\rho, \tilde{\rho})\|v\|_{\tilde{\rho}}}, \frac{\|\mathcal{R} \tilde{v}\|_{\rho-\delta}+\frac{c_{R}(\delta) C_{N}(\rho, \tilde{\rho})}{\gamma \delta^{\tau}}\|v\|_{\tilde{\rho}}}{\|\tilde{v}\|_{\rho}-C_{N}(\rho, \tilde{\rho})\|v\|_{\tilde{\rho}}},\right],
$$

where $c_{R}(\delta)$ is given by Equation (6). 


\section{On the numerical computation of the analytic norm}

Let us describe the method used in this paper to numerically compute the analytic norm of a periodic function of the form

$$
v_{s,\left\{a_{k}\right\}}(\theta)=\sum_{k \in \mathbb{Z}^{n} \backslash\{0\}} \hat{v}_{k} \mathrm{e}^{2 \pi \mathrm{i} k \cdot \theta}, \quad \hat{v}_{k}=a_{k} \frac{\mathrm{e}^{-2 \pi|k|_{1} \hat{\rho}}}{|k|_{1}^{s}}
$$

where $\hat{\rho}>0$, and $a_{k} \in \overline{\mathbb{D}}$. We will denote $v_{s}$ if $a_{k}=1$ for every $k \in \mathbb{Z}^{n} \backslash\{0\}$. We will also denote $v_{s,+}$ if $a_{k}=1$ for every $k \in \mathbb{Z}^{n} \backslash\{0\}$ such that $k_{i} \geq 0$, and $a_{k}=0$ otherwise. These functions are analytic in the complex strip $\mathbb{T}_{\hat{\rho}}^{n}$. Moreover, the Fourier coeficients of $v_{s,\left\{a_{k}\right\}}$ decay as $\left|a_{k}\right||k|^{-s}$ at the boundary of $\mathbb{T}_{\hat{\rho}}^{n}$.

The idea consists in approximating the Fourier series of the considered function using the support $\mathcal{I}_{N}$, given in 22). Given a function $v:=v_{s,\left\{a_{k}\right\}}$, defined for certain $\hat{\rho}>0$, we choose a number $\rho<\hat{\rho}$, and we select a discretization $\mathcal{I}_{N}$ in order to guarantee a precision $\varepsilon$ in the numerical approximation of the norm $\|v\|_{\rho}$. Notice that this discretization will keep the required tolerance when computing $\|v\|_{\rho-\delta}$, for every $0<\delta \leq \rho$.

Remark 2. Notice that one must be very careful with round-off errors. The norm $\|v\|_{\rho}$ is obtained by computing the maximum of the function $v$ restricted to the boundary $\partial \mathbb{T}_{\rho}^{n}$. To this end, we must multiply the Fourier coefficients $\hat{v}_{k}$ by exponentials of the form $\mathrm{e}^{ \pm 2 \pi\left(k_{1} \pm \cdots \pm k_{n}\right) \rho}$. Since the coefficients $\hat{v}_{k}$ decay as $\mathrm{e}^{-2 \pi|k|_{1} \hat{\rho}}$, then the mentioned multiplications produce very large round-off errors, specially if $\rho$ is close to $\hat{\rho}$. For this reason, the factors $\mathrm{e}^{-2 \pi|k|_{1} \hat{\rho}}$ are written separately in the definitions of 25 .

In particular, we take a uniform grid $N=(M, \ldots, M)$ where $M=2^{q}$ is the smallest power such that

$$
\frac{\mathrm{e}^{-\pi(\hat{\rho}-\rho) M / 2}}{(M / 4)^{s}}<\varepsilon
$$

Then, we evaluate the function $v=v_{s,\left\{a_{k}\right\}}$ on the boundary $\partial \mathbb{T}_{\rho}^{n}$ thus obtaining $2^{n}$ samples of $\# \mathcal{I}_{N}$ elements, perform the backward fast Fourier transform associated to these $2^{n}$ samples, and select the maximum value from the obtained numbers. This value is a good approximation, with error $\mathcal{O}(1 / M)$, of the maximum of the function at the boundary. Finally, we refine the computation of this maximum using the Newton method, thus obtaining $\|\tilde{v}\|_{\rho}$ the norm associated with the discrete Fourier transform of $v$ in $\mathcal{I}_{N}$. Then, the true norm is enclosed in the interval (see Theorem 2)

$$
\|v\|_{\rho} \in\left[\|\tilde{v}\|_{\rho}-C_{N}(\rho, \tilde{\rho})\|v\|_{\tilde{\rho}},\|\tilde{v}\|_{\rho}+C_{N}(\rho, \tilde{\rho})\|v\|_{\tilde{\rho}}\right]
$$

for $\rho<\tilde{\rho}<\hat{\rho}$. In the following computations, we select the number $\tilde{\rho}$ that minimizes the length of such interval. The norm $\|v\|_{\tilde{\rho}}$ is simply overestimated analytically.

To illustrate the above methodology, we consider the function $v_{s}$, given by (25) with $a_{k}=1$. In addition to the fact that we have an accurate control of the decay of the Fourier coefficients, this family has the property that the norm $\left\|v_{s}\right\|_{\rho}$ can be evaluated explicitly. Our aim is to convince the reader that, even though we consider test functions with infinitely many harmonics, we can choose a suitable discretization providing a good description of the norm with a finite amount of computations. This may seem obvious at a first glance, but we have to take into 
account the effect of the infinitely many small divisors (see Theorem 2 and Corollary 1). All computations have been performed with 30 digits (using the MPFR library [17]).

We first consider the case $n=1$. In the following computations we use the simple observation that a periodic function having real Fourier coefficients all with the same sign attains its maximum at the point $\theta=0$. Then, we compute $\left\|v_{0}\right\|_{\rho}$ for $0 \leq \rho<\hat{\rho}$, as follows:

$$
\left\|v_{0}\right\|_{\rho}=\sum_{k>0} \mathrm{e}^{-2 \pi k(\hat{\rho}-\rho)}+\sum_{k>0} \mathrm{e}^{-2 \pi k(\hat{\rho}+\rho)}=\frac{\cosh (2 \pi \rho)-\mathrm{e}^{-2 \pi \hat{\rho}}}{\cosh (2 \pi \hat{\rho})-\cosh (2 \pi \rho)} .
$$

Notice that $\left\|v_{0}\right\|_{\rho} \rightarrow \infty$ when $\rho \rightarrow \hat{\rho}$. In order to consider the case $\rho=\hat{\rho}$ we assume that $s>1$ and observe that

$$
\left\|v_{s}\right\|_{\rho}=\sum_{k>0} \frac{\mathrm{e}^{-2 \pi k(\hat{\rho}-\rho)}}{k^{s}}+\sum_{k>0} \frac{\mathrm{e}^{-2 \pi k(\hat{\rho}+\rho)}}{k^{s}}=\operatorname{Li}_{s}\left(\mathrm{e}^{-2 \pi(\hat{\rho}-\rho)}\right)+\operatorname{Li}_{s}\left(\mathrm{e}^{-2 \pi(\hat{\rho}+\rho)}\right),
$$

where $\operatorname{Li}_{s}(z)$ is the polylogarithm function. In Table 1 we present some computations of the norm $\left\|v_{0}\right\|_{\rho}$ using the method described. We compare with the exact value of this norm and with the constant $C_{N}(\rho, \tilde{\rho})$ that can be used to rigorously enclose the numerical computations. We ask for a tolerance $\varepsilon=10^{-30}$. The computations saturate the precision of the machine.

\begin{tabular}{|c|cccc|}
\hline$\rho$ & $N$ & $\left\|\tilde{v}_{0}\right\|_{\rho}$ & $\frac{\left\|\tilde{v}_{0}\right\| \rho-\left\|v_{0}\right\| \rho}{\left\|v_{0}\right\|_{\rho}}$ & $C_{N}(\rho, \tilde{\rho})$ \\
\hline 0.0 & 64 & $3.7418731973 \mathrm{e}-03$ & $0.0 \mathrm{e}+00$ & $9.6 \mathrm{e}-88$ \\
0.1 & 64 & $4.5099874933 \mathrm{e}-03$ & $-2.0 \mathrm{e}-30$ & $5.1 \mathrm{e}-79$ \\
0.2 & 64 & $7.1365311745 \mathrm{e}-03$ & $-4.3 \mathrm{e}-31$ & $2.8 \mathrm{e}-70$ \\
0.3 & 64 & $1.2735869952 \mathrm{e}-02$ & $0.0 \mathrm{e}+00$ & $1.5 \mathrm{e}-61$ \\
0.4 & 128 & $2.3749435599 \mathrm{e}-02$ & $0.0 \mathrm{e}+00$ & $3.3 \mathrm{e}-105$ \\
0.5 & 128 & $4.5246411394 \mathrm{e}-02$ & $0.0 \mathrm{e}+00$ & $1.0 \mathrm{e}-87$ \\
0.6 & 128 & $8.8185405159 \mathrm{e}-02$ & $-1.1 \mathrm{e}-30$ & $3.0 \mathrm{e}-70$ \\
0.7 & 256 & $1.7903995865 \mathrm{e}-01$ & $0.0 \mathrm{e}+00$ & $3.8 \mathrm{e}-105$ \\
0.8 & 256 & $3.9785030192 \mathrm{e}-01$ & $-4.9 \mathrm{e}-31$ & $3.8 \mathrm{e}-70$ \\
0.9 & 512 & $1.1435745379 \mathrm{e}+00$ & $-3.4 \mathrm{e}-30$ & $5.9 \mathrm{e}-70$ \\
0.93 & 1024 & $1.8101817456 \mathrm{e}+00$ & $1.3 \mathrm{e}-30$ & $8.9 \mathrm{e}-98$ \\
0.96 & 2048 & $3.4997999963 \mathrm{e}+00$ & $1.3 \mathrm{e}-30$ & $1.5 \mathrm{e}-111$ \\
0.99 & 8192 & $1.5420733666 \mathrm{e}+01$ & $-1.1 \mathrm{e}-29$ & $5.5 \mathrm{e}-111$ \\
0.995 & 16384 & $3.1333610168 \mathrm{e}+01$ & $4.8 \mathrm{e}-30$ & $1.0 \mathrm{e}-110$ \\
0.999 & 65536 & $1.5865547020 \mathrm{e}+02$ & $-4.6 \mathrm{e}-29$ & $1.2 \mathrm{e}-87$ \\
0.9999 & 524288 & $1.5910494868 \mathrm{e}+03$ & $1.5 \mathrm{e}-27$ & $9.3 \mathrm{e}-69$ \\
\hline
\end{tabular}

Table 1: Numerical computation of the analytic norm of the function $v_{0}$ with $n=1$ and $\hat{\rho}=1$, for several values of $\rho$. We show the relative error using the explicit formula and we include also the minimum of $C_{N}(\rho, \tilde{\rho})$ with respect to $\tilde{\rho}$, with $\rho<\tilde{\rho}<\hat{\rho}$.

For the case $n=2$, we restrict to functions $v_{s,+}$, thus simplifying the combinatorics of the computations. We have

$$
\begin{aligned}
\left\|v_{s,+}\right\|_{\rho} & =\sum_{\ell=1}^{\infty} \sum_{k_{1}+k_{2}=\ell} \frac{\mathrm{e}^{-2 \pi \ell(\hat{\rho}-\rho)}}{\ell^{s}} \\
& =\sum_{\ell=1}^{\infty}(\ell+1) \frac{\mathrm{e}^{-2 \pi \ell(\hat{\rho}-\rho)}}{\ell^{s}}=\operatorname{Li}_{s-1}\left(\mathrm{e}^{-2 \pi(\hat{\rho}-\rho)}\right)+\operatorname{Li}_{s}\left(\mathrm{e}^{-2 \pi(\hat{\rho}-\rho)}\right) .
\end{aligned}
$$

Table 2 is analogous to Table 1 for the function $v_{0,+}$ with $n=2$. Of course, the memory cost in the second case is more demanding, so we do not approach the boundary as much as in the first case. 


\begin{tabular}{|c|cccc|}
\hline$\rho$ & $N$ & $\left\|\tilde{v}_{0,+}\right\|_{\rho}$ & $\frac{\left\|\tilde{v}_{0,+}\right\| \rho-\left\|v_{0,+}\right\|_{\rho}}{\left\|v_{0,+}\right\|_{\rho}}$ & $C_{N}(\rho, \tilde{\rho})$ \\
\hline 0.0 & $64 \times 64$ & $3.7453736011 \mathrm{e}-03$ & $0.0 \mathrm{e}+00$ & $1.9 \mathrm{e}-87$ \\
0.1 & $64 \times 64$ & $7.0378103397 \mathrm{e}-03$ & $4.4 \mathrm{e}-31$ & $1.0 \mathrm{e}-78$ \\
0.2 & $64 \times 64$ & $1.3253135843 \mathrm{e}-02$ & $0.0 \mathrm{e}+00$ & $5.6 \mathrm{e}-70$ \\
0.3 & $64 \times 64$ & $2.5059548137 \mathrm{e}-02$ & $-4.9 \mathrm{e}-31$ & $3.1 \mathrm{e}-61$ \\
0.4 & $128 \times 128$ & $4.7753162472 \mathrm{e}-02$ & $5.2 \mathrm{e}-31$ & $7.0 \mathrm{e}-105$ \\
0.5 & $128 \times 128$ & $9.2371351668 \mathrm{e}-02$ & $-5.3 \mathrm{e}-31$ & $2.1 \mathrm{e}-87$ \\
0.6 & $128 \times 128$ & $1.8405377620 \mathrm{e}-01$ & $-5.4 \mathrm{e}-31$ & $7.1 \mathrm{e}-70$ \\
0.7 & $256 \times 256$ & $3.9008106334 \mathrm{e}-01$ & $-1.0 \mathrm{e}-31$ & $1.0 \mathrm{e}-104$ \\
0.8 & $256 \times 256$ & $9.5395121039 \mathrm{e}-01$ & $1.2 \mathrm{e}-30$ & $1.3 \mathrm{e}-69$ \\
0.9 & $512 \times 512$ & $3.5948837747 \mathrm{e}+00$ & $0.0 \mathrm{e}+00$ & $3.9 \mathrm{e}-69$ \\
0.93 & $1024 \times 1024$ & $6.8970910155 \mathrm{e}+00$ & $-2.2 \mathrm{e}-30$ & $8.2 \mathrm{e}-97$ \\
0.96 & $2048 \times 2048$ & $1.9248159655 \mathrm{e}+01$ & $2.6 \mathrm{e}-30$ & $2.4 \mathrm{e}-110$ \\
\hline
\end{tabular}

Table 2: Numerical computation of the analytic norm of the function $v_{0,+}$ with $n=2$ and $\hat{\rho}=1$, for several values of $\rho$. Implementation details are the same as in Table 1

In Table 3 we present some computations of the norm $\left\|v_{s}\right\|_{\rho}$ at the boundary, i.e. taking $\rho=\hat{\rho}$. We use the same implementation parameters as before. We consider the cases $n=1$ and $n=2$ in the same table. The numbers illustrate the dependence of the computational cost on the regularity of the function. Notice that controlling the tail using $C_{N}(\rho, \rho)$ does not make sense. To this end, it is not difficult to extend the arguments in Section 2.3 to consider $\mathcal{C}^{r}$ functions. In this case, one uses that the decay of Fourier coefficients of a $\mathcal{C}^{r}$-function is of the form $\left|\hat{f}_{k}\right| \leq(2 \pi k)^{-r}\|f\|_{\mathcal{C}^{r}}$.

\begin{tabular}{|c|ccc|ccc|}
\hline & \multicolumn{3}{|c|}{$n=1$} & \multicolumn{2}{c|}{$n=2$} \\
\hline$s$ & $N$ & $\left\|\tilde{v}_{s}\right\|_{\rho}$ & $\frac{\left\|\tilde{v}_{s}\right\|_{\rho}-\left\|v_{s}\right\|_{\rho}}{\left\|v_{s}\right\|_{\rho}}$ & $N$ & $\left\|\tilde{v}_{s,+}\right\|_{\rho}$ & $\frac{\left\|\tilde{v}_{s,+}\right\| \rho-\left\|v_{s,+}\right\|_{\rho}}{\| v_{s,+}} \| \rho$ \\
\hline 15 & 512 & $1.0000340756 \mathrm{e}+00$ & $0.0 \mathrm{e}+00$ & $512 \times 512$ & $2.0000918364 \mathrm{e}+00$ & $-1.5 \mathrm{e}-30$ \\
14 & 1024 & $1.0000647355 \mathrm{e}+00$ & $0.0 \mathrm{e}+00$ & $1024 \times 1024$ & $2.0001839615 \mathrm{e}+00$ & $0.0 \mathrm{e}+00$ \\
13 & 1024 & $1.0001262007 \mathrm{e}+00$ & $-7.8 \mathrm{e}-31$ & $1024 \times 1024$ & $2.0003687999 \mathrm{e}+00$ & $-2.5 \mathrm{e}-29$ \\
12 & 2048 & $1.0002495739 \mathrm{e}+00$ & $7.8 \mathrm{e}-31$ & $2048 \times 2048$ & $2.0007402752 \mathrm{e}+00$ & $-7.0 \mathrm{e}-30$ \\
11 & 4096 & $1.0004976759 \mathrm{e}+00$ & $7.8 \mathrm{e}-31$ & & & \\
10 & 4096 & $1.0009980625 \mathrm{e}+00$ & $-8.8 \mathrm{e}-29$ & & & \\
9 & 16384 & $1.0020118802 \mathrm{e}+00$ & $-2.3 \mathrm{e}-30$ & & & \\
8 & 32768 & $1.0040808435 \mathrm{e}+00$ & $-5.7 \mathrm{e}-29$ & & & \\
7 & 131072 & $1.0083527647 \mathrm{e}+00$ & $-1.3 \mathrm{e}-28$ & & & \\
6 & 524288 & $1.0173465493 \mathrm{e}+00$ & $-5.0 \mathrm{e}-27$ & & & \\
\hline
\end{tabular}

Table 3: Numerical computation of the analytic norm at $\rho=\hat{\rho}$ of the function $v_{s}$ with $\hat{\rho}=1$, for several values of $s$. We show the relative error using the explicit formula.

We observe from Tables 1, 2 and 3 that the accuracy of the computation when the required number of Fourier coefficients becomes too large. This fact is probably related to the fact that the FFT algorithm does not minimize the round-off errors.

\section{Numerical experiments}

Along this section we present several numerical explorations to illustrate the sharpness of the inequalities in (15). Since $F_{\rho, \delta, \omega} v$ depends on multiple parameters, we organize the computations in different subsections. All computations in this section have been performed with 30 digits. 

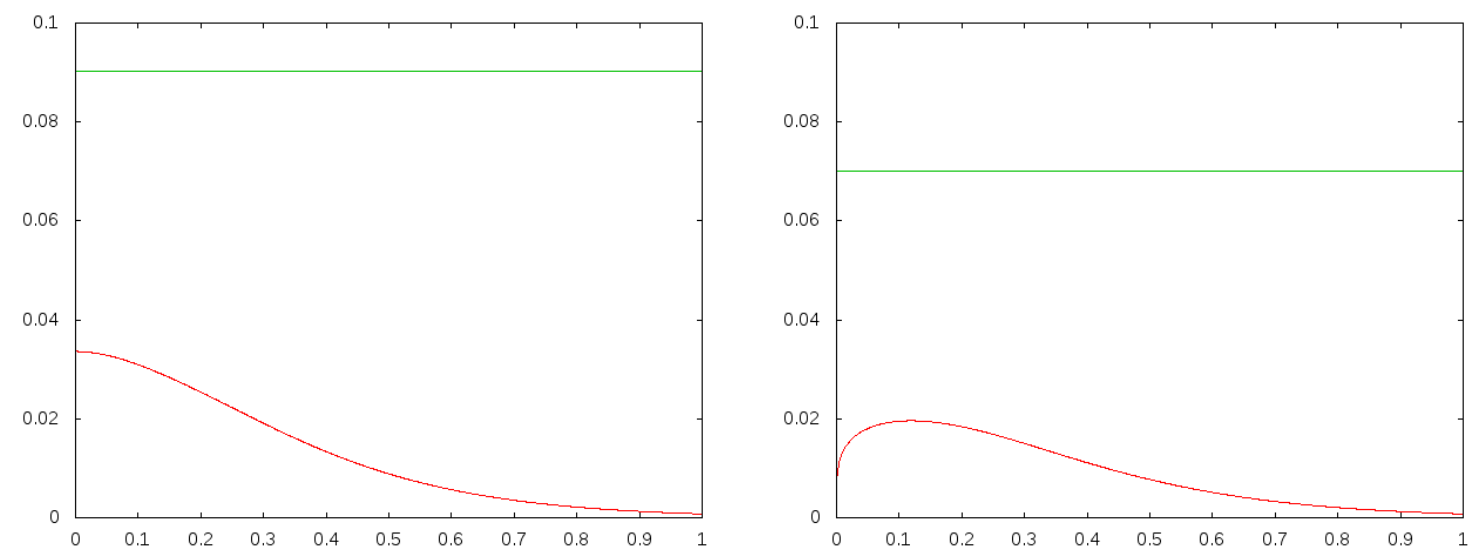

Figure 1: Left $(\tau=1)$ and right ( $\tau=1.2$ ) plots: we show the curve $\delta \mapsto c_{R}(\delta)$ (in red), and the constants $c_{R}^{0}$ (in green).

\subsection{The case of the golden number}

Here we consider the special case where $\omega=\frac{\sqrt{5}-1}{2}$ is the golden mean. It is well known (see e.g. [6]) that this number has Diophantine constants

$$
\gamma=\frac{3-\sqrt{5}}{2}, \quad \tau=1
$$

In Figure 1 we show the values of $c_{R}(\delta)$ and $c_{R}^{0}$ associated to this number. In the left plot we use the value $\tau=1$ and observe how $c_{R}(\delta)$ improves the bound given by the classic constant $c_{R}^{0}$. In the right plot we compare $c_{R}(\delta)$ and $c_{R}^{0}$ using $\tau=1.2$. This choice is very interesting from the KAM point of view since it ensures a positive measure of Diophantine numbers in a neighborhood of $\omega$. In this case, we observe that the improvement of ad hoc estimates $c_{R}(\delta)$ increases when $\delta$ is small.

In order to quantify how good are the Rüssmann estimates for a given function, we consider now the function $v_{0}$, given by Equation (25) with $a_{k}=1$ for every $k \neq 0$, and $\hat{\rho}=1$. Given a value $\rho<1$, we consider the values of $\delta=\frac{j}{100} \rho$, for $j=1 \div 100$, and compute $F_{\rho, \delta, \omega} v_{0}$ and $c_{R}(\delta)$. In Figure 2 we show the overestimation of the two inequalities in (15), using $\log _{10}$-scale. In all computations we use $\tau=1$. The red curve corresponds to

$$
\delta \longmapsto I_{R}:=\frac{c_{R}(\delta)}{\gamma \delta} \frac{1}{F_{\rho, \delta, \omega} v_{0}},
$$

which stands for the overestimation produced by the ad hoc Rüssmann estimates. The overestimation produced by classic Rüssmann estimates is given by the curve

$$
\delta \longmapsto \frac{c_{R}^{0}}{\gamma \delta} \frac{1}{F_{\rho, \delta, \omega} v_{0}},
$$

which is plotted in green. The results show that the use of ad hoc estimates $c_{R}(\delta)$ outperforms the classic bound $c_{R}^{0}$. In some cases by several orders of magnitude. We observe the following:

- If $\rho$ is far from $\hat{\rho}$, the Fourier coefficients of both $v_{0}(\theta \pm \mathrm{i} \rho)$ and $\mathcal{R} v_{0}(\theta \pm \mathrm{i}(\rho-\delta))$ decay very fast, and the effect of the high order small divisors is negligible. For this reason, 

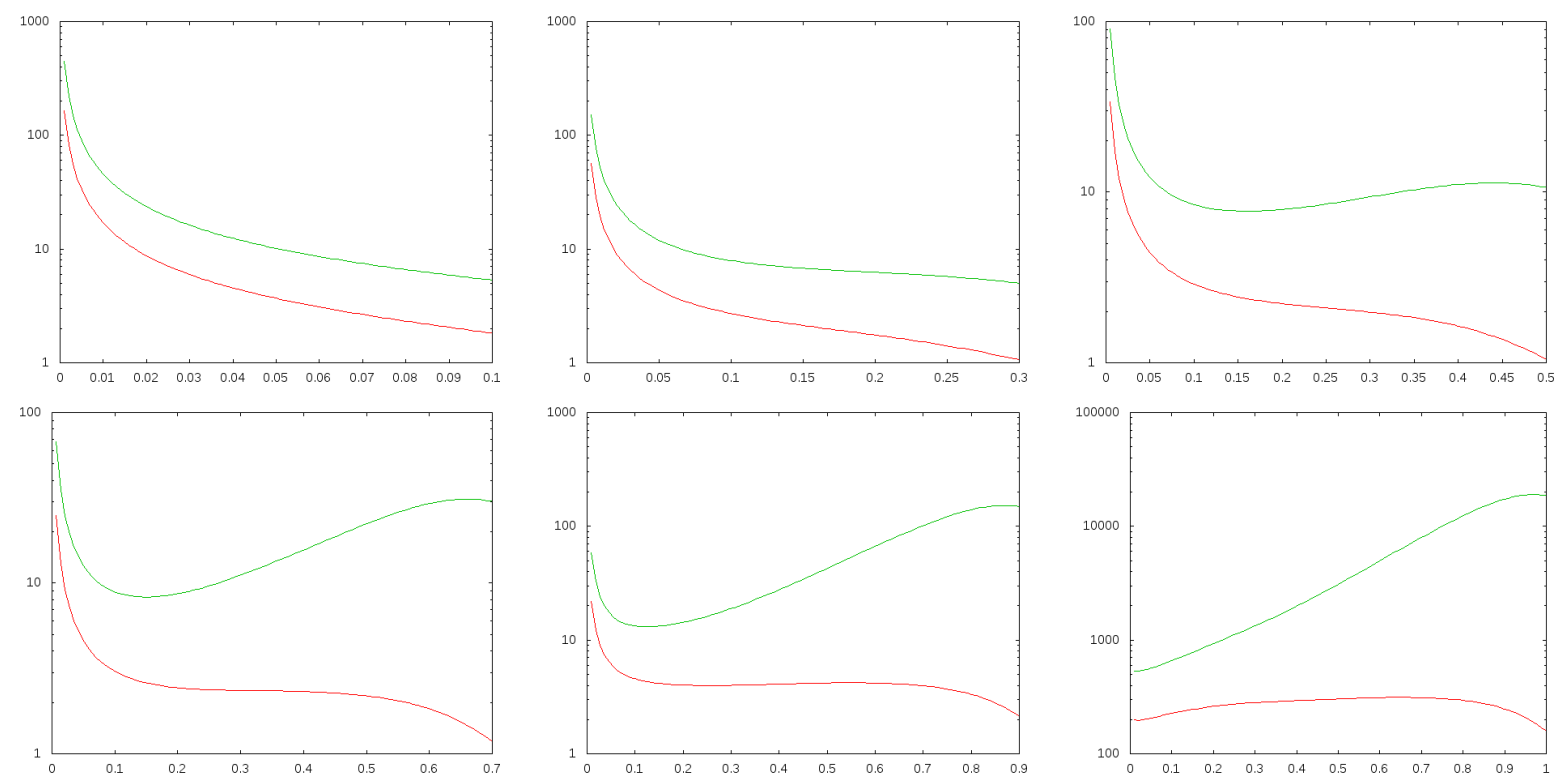

Figure 2: Overestimation (in $\log _{10}$-scale) of the Rüssmann estimates ( $y$-axis) versus $\delta$ ( $x$-axis). We consider the golden rotation and the function $v_{0}$ with $\hat{\rho}=1$. Every plot corresponds to a different value of $\rho$ in $\{0.1,0.3,0.5,0.7,0.9,0.999\}$. The red curve corresponds to [26, which is the overestimation using ad hoc estimates. The green curve corresponds to 27).
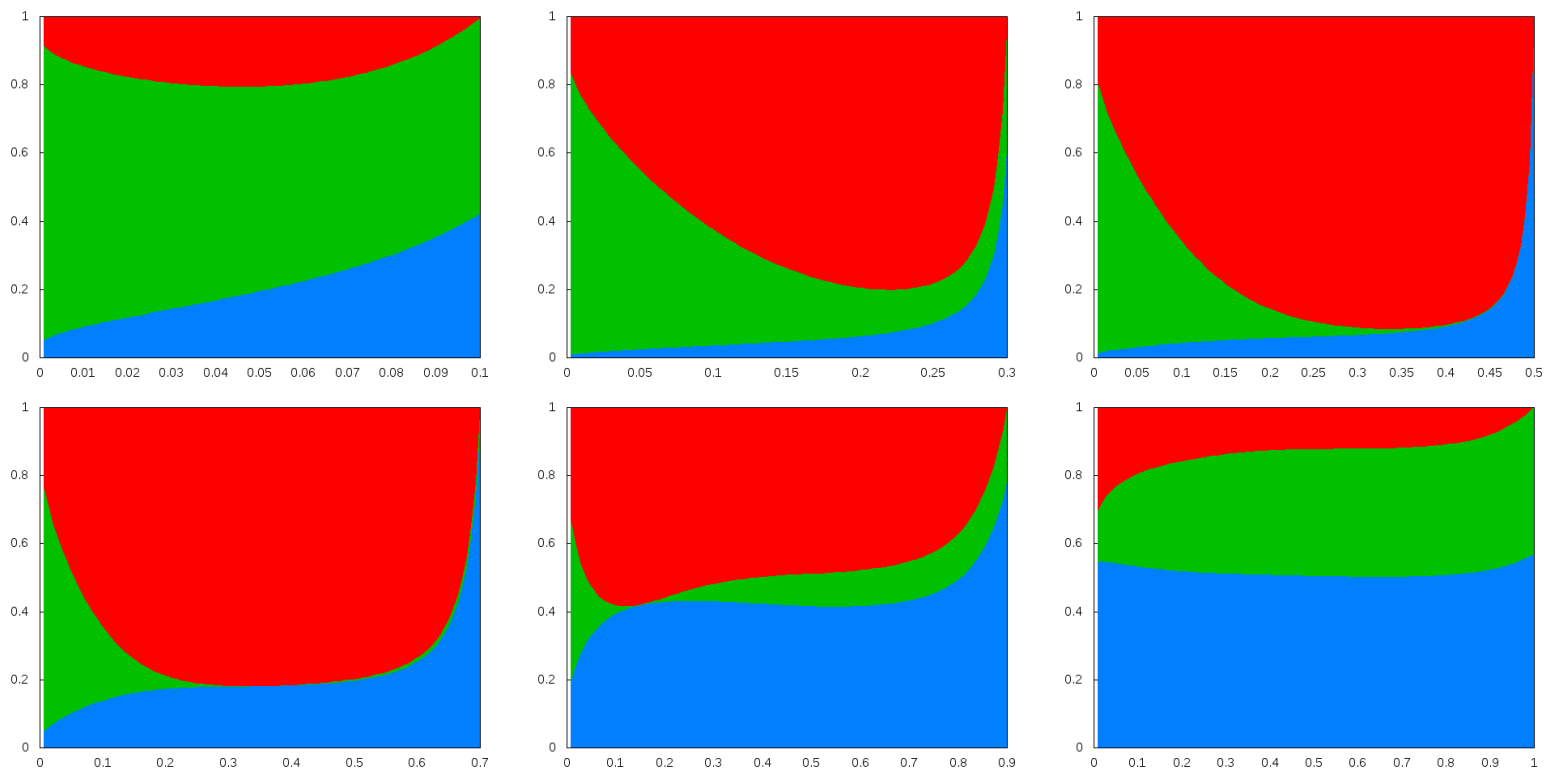

Figure 3: Fraction (in logarithmic scale) of the contribution of different error sources $I_{1}, I_{2}$, and $I_{3}$ ( $y$-axis) versus $\delta$ ( $x$-axis); given by (17), [18), and (19), respectively. We consider the golden rotation and the function $v_{0}$ with $\hat{\rho}=1$. Every plot corresponds to a different value of $\rho$ in $\{0.1,0.3,0.5,0.7,0.9,0.999\}$. The red area is $\frac{\log \left(I_{1}\right)}{\log \left(I_{R}\right)}$, the green area is $\frac{\log \left(I_{2}\right)}{\log \left(I_{R}\right)}$, the blue area is $\frac{\log \left(I_{3}\right)}{\log \left(I_{R}\right)}$.

we observe a large overestimation for small values of $\delta$ in all cases (see the discussion in Remark 1). 
- The best results are obtained for intermediate values of $\rho$ (that is $\rho \in(0.3,0.8)$ and not very small values of $\delta$ (say $\delta \in(\rho / 5, \rho]$ ). In these cases, we have $I_{R} \in(1,4]$. We also observe that if $0 \ll \delta \approx \rho$ the ad hoc Rüssmann estimates provide a sharp upper bound: $F_{\rho, \delta, \omega} v \approx c_{R}(\delta) \gamma^{-1} \delta^{-\tau}$.

- If $\rho$ approaches $\hat{\rho}$, then the performance of the Rüssmann estimates deteriorates. This is because the factor (20) becomes very large. For example, according to Table 1 , we have $\left\|v_{0}\right\|_{0.999} \approx 159$ and $\left\|\mathcal{R} v_{0}\right\|_{0}$ is small.

In Figure 3 we break down $I_{R}$ into the sources of overestimation $I_{1}, I_{2}$, and $I_{3}$ of the ad hoc Rüssmann estimates. These charts allows us to observe, in case that the overestimation is significant, which is the relative contribution of each factor.

\begin{tabular}{|c|cccccccccccccc|}
\hline$\rho$ & 0.1 & 0.2 & 0.3 & 0.4 & 0.5 & 0.6 & 0.7 & 0.8 & 0.9 & 0.93 & 0.96 & 0.99 & 0.993 & 0.999 \\
$\delta$ & 0.02 & 0.04 & 0.06 & 0.08 & 0.1 & 0.12 & 0.14 & 0.16 & 0.18 & 0.186 & 0.192 & 0.198 & 0.1986 & 0.1998 \\
Eq. [26] & 8.7 & 5.0 & 3.8 & 3.2 & 2.9 & 2.7 & 2.7 & 2.9 & 4.1 & 5.1 & 7.9 & 27.4 & 38.5 & 261.3 \\
Eq. [27] & 23.6 & 13.6 & 10.6 & 9.2 & 8.5 & 8.1 & 8.3 & 9.4 & 13.8 & 17.7 & 27.6 & 96.9 & 136.6 & 929.8 \\
$I_{1}$ & 1.47 & 1.78 & 1.93 & 2.00 & 2.02 & 2.02 & 2.03 & 2.09 & 2.22 & 2.27 & 2.33 & 2.39 & 2.40 & 2.41 \\
$I_{2}$ & 4.61 & 2.58 & 1.91 & 1.57 & 1.37 & 1.23 & 1.12 & 1.03 & 1.01 & 1.05 & 1.20 & 2.03 & 2.38 & 6.07 \\
$I_{3}$ & 1.29 & 1.09 & 1.04 & 1.03 & 1.05 & 1.09 & 1.17 & 1.34 & 1.81 & 2.15 & 2.83 & 5.64 & 6.74 & 17.84 \\
\hline$\rho$ & 0.1 & 0.2 & 0.3 & 0.4 & 0.5 & 0.6 & 0.7 & 0.8 & 0.9 & 0.93 & 0.96 & 0.99 & 0.993 & 0.999 \\
$\delta$ & 0.1 & 0.2 & 0.3 & 0.4 & 0.5 & 0.6 & 0.7 & 0.8 & 0.9 & 0.93 & 0.96 & 0.99 & 0.993 & 0.999 \\
Eq. 26] & 1.8 & 1.2 & 1.1 & 1.0 & 1.0 & 1.1 & 1.2 & 1.4 & 2.1 & 2.8 & 4.5 & 16.4 & 23.2 & 159.7 \\
Eq. [27] & 5.3 & 4.2 & 5.0 & 7.0 & 10.7 & 17.4 & 30.2 & 58.7 & 150.1 & 229.9 & 430.5 & 1839.4 & 2644.8 & 18754.4 \\
$I_{1}$ & 1.00 & 1.00 & 1.00 & 1.00 & 1.00 & 1.00 & 1.00 & 1.00 & 1.00 & 1.00 & 1.00 & 1.00 & 1.00 & 1.00 \\
$I_{2}$ & 1.41 & 1.09 & 1.02 & 1.00 & 1.00 & 1.00 & 1.01 & 1.04 & 1.18 & 1.30 & 1.59 & 2.90 & 3.44 & 8.93 \\
$I_{3}$ & 1.29 & 1.09 & 1.04 & 1.03 & 1.05 & 1.09 & 1.17 & 1.34 & 1.81 & 2.15 & 2.83 & 5.64 & 6.74 & 17.84 \\
\hline
\end{tabular}

Table 4: Some selected computations of the Rüssmann estimates. We consider the golden rotation and the function $v_{0}$ with $\hat{\rho}=1$. The upper table corresponds to $\delta=\frac{\rho}{5}$ and the lower table corresponds to $\delta=\rho$.

Table 4 reflects quantitatively the above observations. In the upper table, we take different values of $\rho$ and values of $\delta$ of the form $\delta=\frac{\rho}{5}$, which corresponds to a choice that is typical when applying the KAM theorem. For $\rho \in(0.3,0.8)$ we observe that the ad hoc Rüssmann estimates only overestimate the norm of $\left\|\mathcal{R} v_{0}\right\|_{\rho-\delta}$ by a factor at most 4 (typically, smaller than 3 ). The lower table corresponds to the same values of $\rho$, but we take $\delta=\rho$. This illustrates the limit case where ad hoc estimates tend to be sharp (for intermediate values of $\rho$ ) and the difference with respect to the classic estimates is larger.

Remark 3. Using other functions we have obtained analogous plots as in Figure 2. In particular, we have considered a collection of $10^{5}$ functions $v_{s,\left\{a_{k}\right\}}$, given by Equation [25), with $\hat{\rho}=1$ and (uniform) random numbers $a_{k} \in \overline{\mathbb{D}}$. If one reproduces Figure 3 for the average behavior of these functions, then the obtained plots are almost identical to the ones obtained for $v_{0}$. Moreover, if we increase the value of the parameter $s$ we observe that the good performance of ad hoc Rüssmann estimates for $0 \ll \delta \approx \rho$ prevails when $\rho \approx \hat{\rho}$ (notice that $\left\|v_{s}\right\|_{\hat{\rho}}$ is finite for $s>1)$. These figures are omitted in order to avoid an unnecessary lengthy paper. 

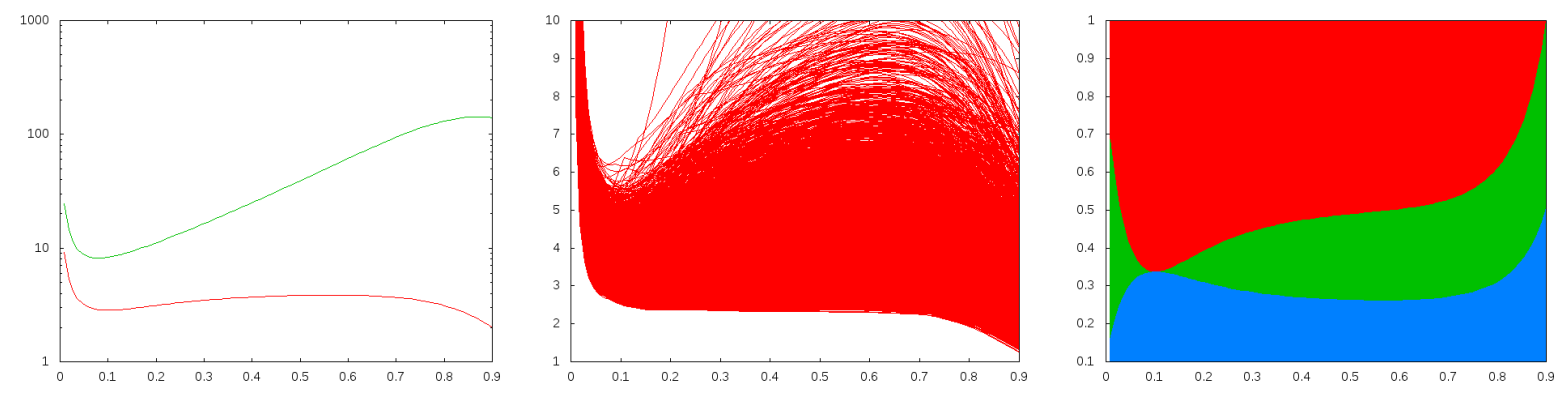

Figure 4: We take $\rho=0.9$ in all plots. Left plot: Overestimation (in $\log _{10}$-scale) of the Rüssmann estimates ( $y$-axis) versus $\delta$ ( $x$-axis). We consider the function $v_{\left\{b_{k}\right\}}$, given by (28) with $b_{k}=1$ and $\hat{\rho}=1$. The red curve corresponds to 26 and the green curve corresponds to 27). Center plot: Overestimation (in normal scale) of the ad hoc Rüssmann estimates ( $y$-axis) versus $\delta$ ( $x$-axis). We consider $10^{5}$ function given by 25 with $\hat{\rho}=1$, and $10^{5}$ function given by (28) with $\hat{\rho}=1$. Right plot: Average fraction (in logarithmic scale) of the contribution of different error sources associated to the center plot (see Figure 3 for color description).

Remark 4. Observe that we are dealing with functions with an infinite number of Fourier coefficients but with an explicit control of their decay. It is worth mentioning what happens when one perturbs these functions by including eventually a large Fourier coefficient. For example, by considering functions of the form

$$
v_{s,\left\{a_{k}\right\}}(\theta)=\sum_{k \in \mathbb{Z}^{n} \backslash\{0\}} \hat{v}_{k} \mathrm{e}^{2 \pi \mathrm{i} k \cdot \theta}+\sum_{k \in \mathcal{I}_{N} \backslash\{0\}} \hat{w}_{k} \mathrm{e}^{2 \pi \mathrm{i} k \cdot \theta}, \quad \hat{v}_{k}=a_{k} \frac{\mathrm{e}^{-2 \pi|k|_{1} \hat{\rho}}}{|k|_{1}^{s}}, \quad \hat{w}_{k} \in \overline{\mathbb{D}} .
$$

In this situation, one observes a poor performance of the Rüssmann estimates: $F_{\rho, \delta, \omega} v \ll$ $c_{R}(\delta) \gamma^{-1} \delta^{-\tau}$. A heuristic justification of this observation follows from the computation in (13).

Finally, we suitably choose a family of functions that are selected to saturate the CauchySchwarz inequality in Equation (8). We consider functions of the form

$$
v_{\left\{b_{k}\right\}}(\theta)=\sum_{k \in \mathbb{Z}^{n} \backslash\{0\}} \hat{v}_{k} \mathrm{e}^{2 \pi \mathrm{i} k \cdot \theta}, \quad \hat{v}_{k}=\frac{b_{k}}{\sin (\pi \omega \cdot k)} \mathrm{e}^{-2 \pi|k|_{1} \hat{\rho}}, \quad b_{k} \in \overline{\mathbb{D}} .
$$

Notice that this family satisfies $I_{2}=1$ when we select $0<\delta \leq \rho<\hat{\rho}$ such that $\hat{\rho}=\rho+\delta$. In the left plot of Figure 4 we reproduce the computations presented in Figures 2 and 3 for the function (28) taking $b_{k}=1$ for every $k$. We observe a behavior that is very close to the Family (25). In the center plot of Figure 4 we show the overestimation produced by the ad hoc Rüssmann estimates for $10^{5}$ functions of Family (25) with (uniform) random numbers $a_{k} \in$ $\overline{\mathbb{D}}$ and $10^{5}$ functions of Family $(28)$ with (uniform) random numbers $b_{k} \in \overline{\mathbb{D}}$. In the range $\delta \in\left[\frac{\rho}{4.5}, \frac{\rho}{1.5}\right]$ we observe that $\min _{v} I_{R} \approx 2.3$. Finally, in the right plot Figure 4 we show average behavior (including all the selected functions) of the contribution of each source of overestimation.

\subsection{Dependence on $\omega$}

Let us consider now the dependence of the Rüssmann estimates on $\omega$. We select the numbers

$$
\omega=\sin \left(\frac{0.02+0.5 j}{10000}\right), \quad \text { for } j=1 \div 10^{4} .
$$


These numbers behave like Diophantine numbers and are eventually "close" to low order resonances. In order to associate a pair $(\gamma, \tau)$ for each value of $\omega$ we use two different approaches:

Method 1: Approximate $\omega$ by a quadratic number $\omega_{Q}$ as follows

$$
\omega \simeq \omega_{Q}=\left[a_{0}, a_{1}, \ldots, a_{Q}, 1,1,1, \ldots\right]=\left[a_{0}, a_{1}, \ldots, a_{Q}, 1^{\infty}\right],
$$

where $a_{i}$ are obtained computing the truncated continued fraction of $\omega$. Then we take $\tau=1$ and compute the constant $\gamma$ associated to $\omega_{Q}$ (see details in [6, Appendix B]).

Method 2: We enclose $\omega$ into a tiny interval and we assign to this interval a pair $(\gamma, \tau)$ that ensures that the relative measure of $(\gamma, \tau)$-Diophantine numbers is positive. We refer to [10, Section 4.1] for details.

We first restrict the analysis to the function $v_{0}$, given by Equation (25) with $a_{k}=1$ for every $k \neq 0$, and we fix the parameters $\delta=0.1, \rho=0.5$, and $\hat{\rho}=1$. We have obtained analogous results for other functions of the family (selecting $a_{k}$ randomly). In Figure 5 we plot

$$
\omega \longmapsto I_{R}:=\frac{c_{R}(\delta)}{\gamma \delta^{\tau}} \frac{1}{F_{\rho, \delta, \omega} v_{0}}
$$

in red (first and second plots), and

$$
\omega \longmapsto \frac{c_{R}^{0}}{\gamma \delta^{\tau}} \frac{1}{F_{\rho, \delta, \omega} v_{0}}
$$

in green (first plot). It is worth mentioning that 29) seems to be a curve which is regular in the sense of Whitney. Moreover, this curve is independent of the values of $(\gamma, \tau)$ if $L$ is taken large enough. On the contrary, the classic estimates present a "bad" dependence on $\omega$, in the sense that they produce a large overestimation quite often (between 100 and 10000 times larger). Let us compare also the sensitivity of classic Rüssmann estimates with respect to the method used to obtain $(\gamma, \tau)$. In general, Method 2 provides better estimates than Method 1, capturing also the effect of resonances.

Analogous computations are presented in Figure 6. In this case, we consider $10^{4}$ functions of the form (28) by taking (uniform) random numbers $b_{k} \in \overline{\mathbb{D}}$. For each value of $\omega$, we solve the cohomological equations and obtain the Rüssmann estimates for all these functions. We have obtained similar results for all the considered functions. In the plots we show the average behavior observed. The results are analogous to the ones obtained for the function $v_{0}$ given by (25), the only remarkable difference being the behavior observed for "bad" Diophantine numbers (i.e. close to resonances). In this case, due to the particular relationship between the size of Fourier coefficients and the small divisors, the improvement of the ad hoc Rüssmann estimates becomes even larger.

In Figure 7 we show the contribution of the different sources of error in the previous computations. This illustrates that the relative importance of $I_{1}, I_{2}$ and $I_{3}$ depends on the family of functions under consideration. As it was discussed in Section 4.1, the difference is not significant when $\omega$ is the golden mean. But Figure 7 shows that we can observe different scenarios for other values of $\omega$. For example, we find that when $\omega$ is close to a resonance the overestimation $I_{2}$ is dominant in the left plot and irrelevant in the right plot. 

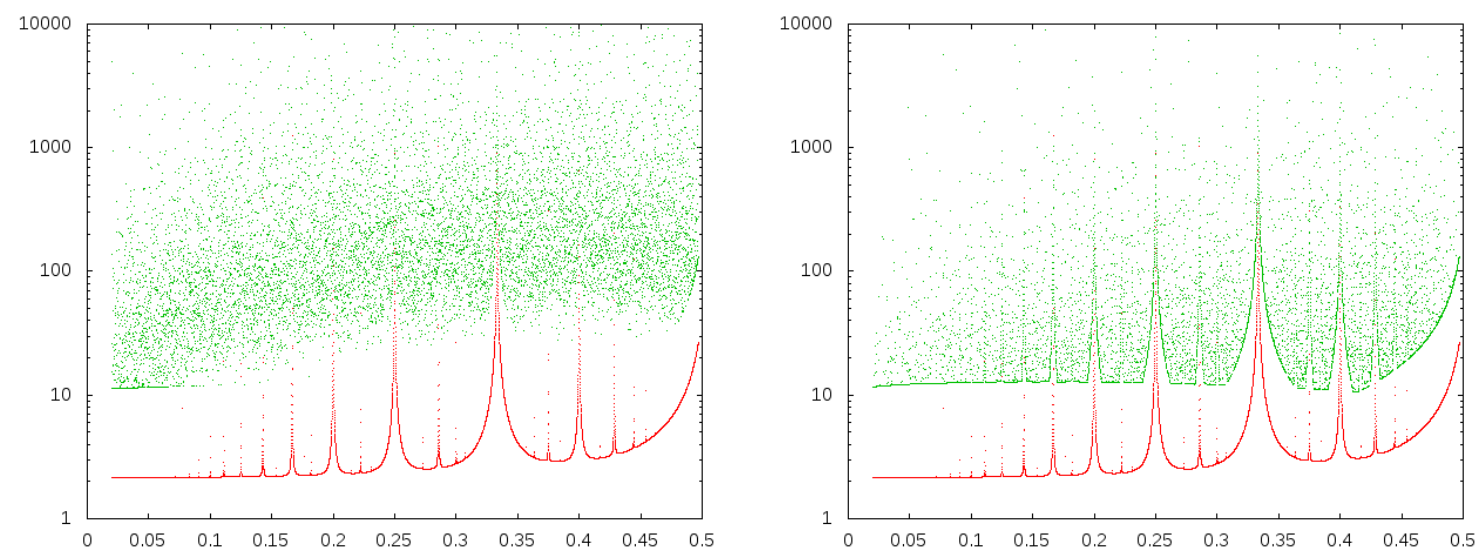

Figure 5: : Overestimation (in $\log _{10}$-scale) of the Rüssmann estimates ( $y$-axis) versus $\omega$ ( $x$-axis). We consider the function $v_{0}$, given by (25) with $\hat{\rho}=1$. We take $\rho=0.5$ and $\delta=0.1$ in all plots. We show (29) in red and (30) in green. In the left plot we use Method 1 and in the right plot we use Method 2.
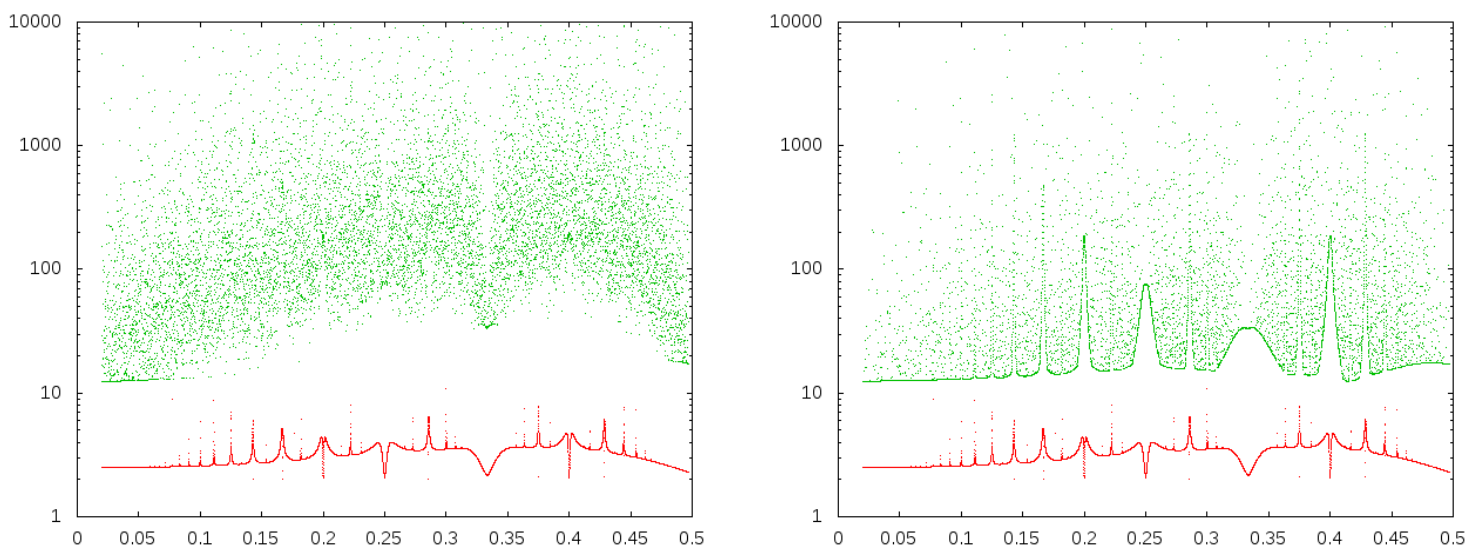

Figure 6: : Overestimation (in $\log _{10}$-scale) of the Rüssmann estimates ( $y$-axis) versus $\omega$ ( $x$-axis). We consider $10^{4}$ function $v_{\left\{b_{k}\right\}}$, given by (28) with $\hat{\rho}=1$ and random numbers $b_{k} \in \overline{\mathbb{D}}$. We take $\rho=0.5$ and $\delta=0.1$ in all plots. We show (29) in red and (30) in green. In the left plot we use Method 1 and in the right plot we use Method 2.

\subsection{Two dimensional case}

Finally, we present some computations to quantify the performance of Rüssmann estimates in the case $n=2$. Now we consider frequency vectors of the form $\omega=\left(\omega_{1}, \omega_{2}\right)$, with

$$
\omega_{1}=\frac{\sqrt{5}-1}{2}, \quad \omega_{2}=\sin \left(\frac{0.02+0.5 j}{10000}\right), \quad \text { for } j=1 \div 10^{4}
$$

The behavior of the estimates agrees qualitatively with the results previously discussed. Hence, in order to reduce the exposition, here we only consider the study of the function $v_{0}$ for the fixed choice of parameters $\rho=0.9$ and $\delta=0.18$. Our aim is to illustrate quantitatively the dependence on $\omega$ when the dimension increases.

The left plot of Figure 8 is analogous to the right plot of Figure 5. In this case, in order to obtain constants $(\gamma, \tau)$ associated to $\omega$ we use Method 2, since Method 1 is not valid (there is no 

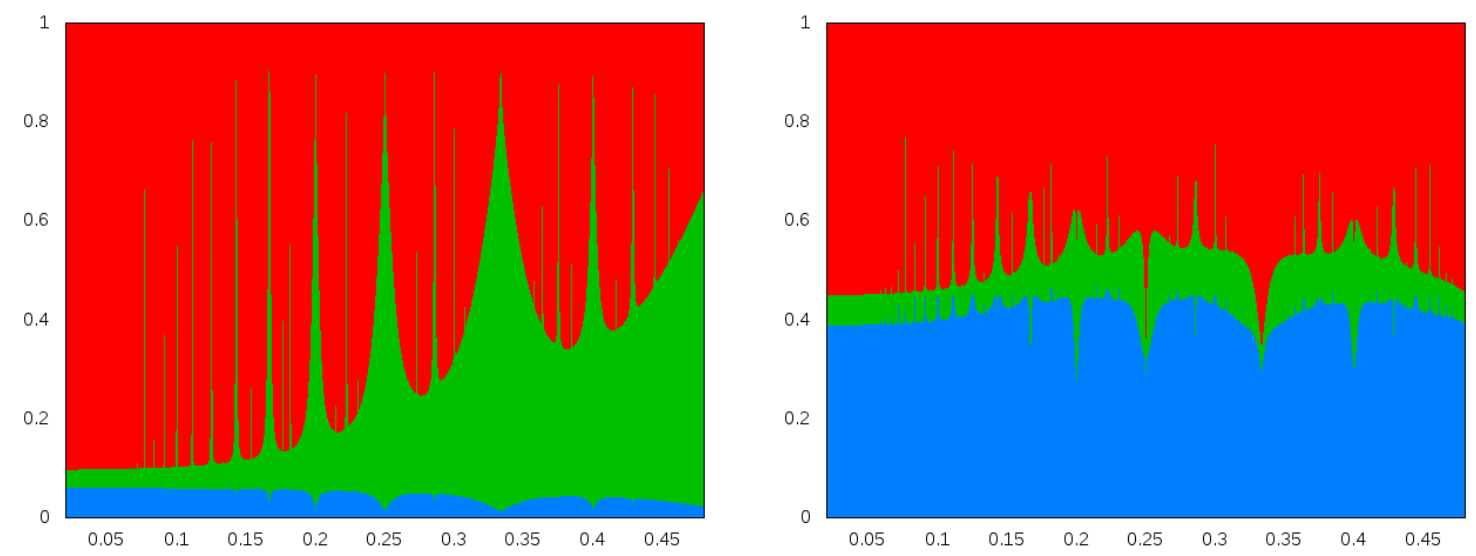

Figure 7: : Average fraction (in logarithmic scale) of the contribution of different error sources $I_{1}, I_{2}$, and $I_{3}$ ( $y$-axis) versus $\delta$ ( $x$-axis) to the overestimation produced the ad hoc Rüssmann estimates. Left plot corresponds to computations in Figure 5 and right plot corresponds to computations in Figure 6. See Figure 3 for color description.
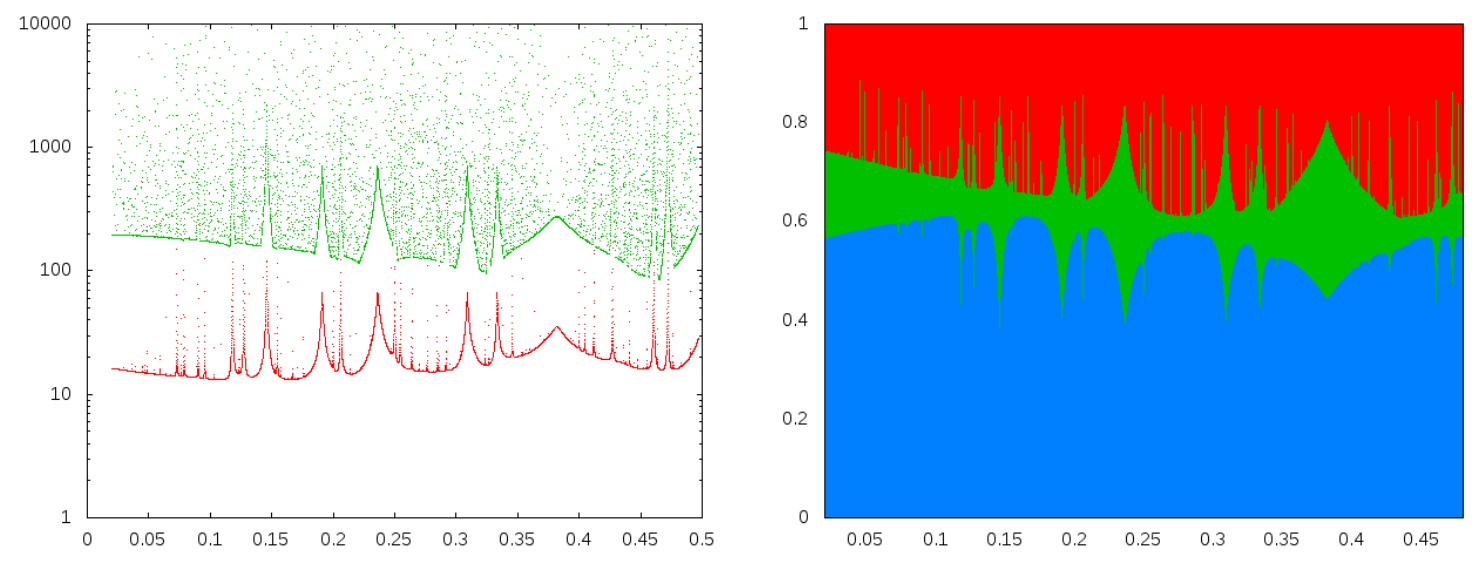

Figure 8: : Left plot: Overestimation (in $\log _{10}$-scale) of the Rüssmann estimates ( $y$-axis) versus $\omega_{2}$ ( $x$-axis) for fixed $\omega_{1}$ ). We consider the function $v_{0}$, given by (25) with $\hat{\rho}=1, \rho=0.9$ and $\delta=0.18$. Right plot: contribution (in logarithmic scale) of different error sources $I_{1}, I_{2}$, and $I_{3}$ ( $y$-axis) versus $\delta$ ( $x$-axis) to the overestimation produced by the ad hoc Rüssmann estimates in the computation of the left plot.

continuous fraction expansion). Again, we observe that $a d$ hoc Rüssmann estimates depend in a nice way on the frequency. The red points seems to be on a curve which has some regularity in the sense of Whitney. The overestimation of classic estimates with respect to the ad hoc ones is quite equivalent as in the one dimensional case. However, we observe that the overestimation of the true norm produced by both estimates is significantly larger, at least a factor 10 .

In the right plot Figure 8 we show the three sources of overestimation. Notice that the blue area (corresponding to $I_{3}$ ) is larger that the blue area observed in the left plot of Figure 7, and the main responsible for the overestimation with respect to the case $n=1$. 


\section{Final remarks and conclusions}

Here we summarize some observations and questions that arise after the presentation of the previous results.

- We observe that if $0 \ll \delta \approx \rho$ and intermediates values of $\rho$, the ad hoc Rüssmann estimates provide a sharp upper bound: $F_{\rho, \delta, \omega} v \approx c_{R}(\delta) \gamma^{-1} \delta^{-\tau}$. This can be readily justified by observing that the three expressions $I_{1}, I_{2}$, and $I_{3}$ tend to saturate in this range of parameters. Furthermore, in Figure 2 we observe that, at this range of parameters, the overestimation $\delta \mapsto I_{R}(\delta)$ seems to be proportional to $\rho-\delta$, as $\delta \rightarrow \rho$. It is of interest to know if this remains true for typical functions.

- It is clear that the three sources of overestimation $I_{1}, I_{2}$, and $I_{3}$ are sharp independently, but in general they are not sharp jointly. For example, $I_{1}$ is saturated by functions with just one harmonic or, if $n=1$, by functions with positive Fourier coefficients only supported at $k>0 ; I_{2}$ is saturated by functions of the form (28) when $\rho+\delta=\hat{\rho}$; and $I_{3}$ is saturated by functions which are almost constant at the boundary of the complex strip $\mathbb{T}_{\rho}$. It seems not clear how to minimize these three overestimations simultaneously. We have numerically observed that at certain regimes this minimization leads to a minimum overestimation $I_{R} \approx 2.3$ (see right plot in Figure 4 ) for the case $n=1$ and $\omega=\frac{\sqrt{5}-1}{2}$.

- We have analyzed the diferent sources of overestimation $I_{R}=I_{1} I_{2} I_{3}$ when a certain function or family of function is fixed. The contribution of each source to the total overestimation depends on the parameters $\delta, \rho$ and $\hat{\rho}$. When specific regimes of the parameters or subfamilies of functions are considered, this information could be interesting to tailor Theorem 1 to improve the estimates.

- When $\omega$ is a generic Diophantine number, the use of ad hoc Rüssmann estimates outperforms the use of classic estimates by several orders of magnitude (see Section 4.2). Moreover, we observe a nice behavior of the overestimation $I_{R}$ with respect to $\omega$. Actually, it seems to exhibit some regularity (in the sense of Whitney) with respect to $\omega$.

\section{Acknowledgements}

We acknowledge the use of the Lovelace (ICMAT-CSIC) cluster for research computing, cofinanced by the Spanish Ministry of Economy and Competitiveness (MINECO), European FEDER funds and the Severo Ochoa Programme. J.-Ll. F. acknowledges the partial support of Essen, L. and C.-G., for mathematical studies. A. H. acknowledges support from the Spanish grant MTM2015-67724-P, and the Catalan grant 2014-SGR-1145. A. L. acknowledges support from the Severo Ochoa Programme for Centres of Excellence in R\&D (SEV-2015-0554), the Spanish grant MTM2016-76072-P, and the ERC Starting Grant 335079.

\section{References}

[1] V.I. Arnold. Proof of a theorem of A. N. Kolmogorov on the preservation of conditionally periodic motions under a small perturbation of the Hamiltonian. Uspehi Mat. Nauk, 18(5 (113)):13-40, 1963. 
[2] V.I. Arnold. Small denominators and problems of stability of motion in classical and celestial mechanics. Russ. Math. Surveys, 18:85-192, 1963.

[3] H.W. Broer, G.B. Huitema, and M.B. Sevryuk. Quasi-periodic motions in families of dynamical systems. Order amidst chaos. Lecture Notes in Math., Vol 1645. SpringerVerlag, Berlin, 1996.

[4] A. Celletti and L. Chierchia. Construction of Analytic KAM Surfaces and Effective Stability Bounds. Comm. Math. Phys., 118(1):199-161, 1988.

[5] A. Celletti and L. Chierchia. On the stability of realistic three-body problems. Comm. Math. Phys., 186(2):413-449, 1997.

[6] A. Celletti and L. Chierchia. KAM stability and celestial mechanics. Mem. Amer. Math. Soc., 187(878):viii+134, 2007.

[7] R. de la Llave. A tutorial on KAM theory. In Smooth ergodic theory and its applications (Seattle, WA, 1999), volume 69 of Proc. Sympos. Pure Math., pages 175-292. Amer. Math. Soc., Providence, RI, 2001.

[8] R. de la Llave and D. Rana. Accurate strategies for small divisor problems. Bull. Amer. Math. Soc. (N.S.), 22(1):85-90, 1990.

[9] R. de la Llave and D. Rana. Accurate strategies for K.A.M. bounds and their implementation. In Computer aided proofs in analysis (Cincinnati, OH, 1989), volume 28 of IMA Vol. Math. Appl., pages 127-146. Springer, New York, 1991.

[10] J.-Ll. Figueras, A. Haro, and A. Luque. Rigorous computer assisted application of kam theory: a modern approach. To appear in Foundations of Computational Mathematics, DOI: 10.1007/s10208-016-9339-3, 2017.

[11] J.M. Greene. A method for determining a stochastic transition. J. Math. Phys, 20(6):1183$1201,1975$.

[12] A.N. Kolmogorov. On conservation of conditionally periodic motions for a small change in Hamilton's function. Dokl. Akad. Nauk SSSR (N.S.), 98:527-530, 1954. Translated in p. 51-56 of Stochastic Behavior in Classical and Quantum Hamiltonian Systems, Como 1977 (eds. G. Casati and J. Ford) Lect. Notes Phys. 93, Springer, Berlin, 1979.

[13] U. Locatelli. Three-body planetary problem: study of KAM stability for the secular part of the Hamiltonian. Planetary and Space Science, 46(11):1453-1464, 1998.

[14] U. Locatelli and A. Giorgilli. Invariant tori in the secular motions of the three-body planetary systems. Cel. Mech., 78(1):47-74, 2000.

[15] J. Moser. On invariant curves of area-preserving mappings of an annulus. Nachr. Akad. Wiss. Göttingen Math.-Phys. Kl. II, 1962:1-20, 1962.

[16] J. Moser. Convergent series expansions for quasi-periodic motions. Math. Ann., 169:136176, 1967. 
[17] N. Revol and F. Rouillier. Motivations for an arbitrary precision interval arithmetic and the mpfi library. Reliable computing, 11(5):275-290, 2005.

[18] H. Rüssmann. On optimal estimates for the solutions of linear partial differential equations of first order with constant coefficients on the torus. In Dynamical systems, theory and applications (Rencontres, Battelle Res. Inst., Seattle, Wash., 1974), pages 598-624. Lecture Notes in Phys., Vol. 38. Springer, Berlin, 1975.

[19] H. Rüssmann. On a new proof of Moser's twist mapping theorem. In Proceedings of the Fifth Conference on Mathematical Methods in Celestial Mechanics (Oberwolfach, 1975), Part I. Celestial Mech., 14(1):19-31, 1976.

[20] H. Rüssmann. On optimal estimates for the solutions of linear difference equations on the circle. In Proceedings of the Fifth Conference on Mathematical Methods in Celestial Mechanics (Oberwolfach, 1975), Part I. Celestial Mech., volume 14, 1976. 